A.C. Montenegro, A.F. Buitrago

\title{
Nueva era de la anticoagulación Resurgimiento de las clínicas de anticoagulación
}

\author{
New era of anticoagulation \\ Resurgence of anticoagulation clinics
}

La anticoagulación es uno de los tratamientos más ampliamente utilizados y tiene indicaciones que comprometen diversas especialidades. Este tratamiento requiere un control y seguimiento muy cuidadoso para evitar complicaciones que pueden comprometer la vida del paciente. Las clínicas de anticoagulación han surgido como un mecanismo para reducir estas complicaciones. Tienen menor número de hospitalizaciones por año, menor número de consultas al servicio de urgencias y menor número de hemorragias. Adicionalmente se ha demostrado una reducción significativa de costos y una reducción de mortalidad (1).

La terapia anticoagulante requiere una educación exhaustiva e individualizada del paciente y su familia que no puede obtenerse en una consulta habitual debido a las restricciones del sistema de salud en nuestro país $(1,2)$.

La warfarina, la heparina no fraccionada, y las heparinas de bajo peso molecular han representado por muchos años la base de la terapia de anticoagulante. Los anticoagulantes tradicionales afectan a múltiples objetivos en la cascada de la coagulación y tienen ciertas limitaciones que hacen compleja su utilización en el ámbito clínico. Los esfuerzos recientes se han centrado en el desarrollo de anticoagulantes con objetivos más específicos tales como los inhibidores del Factor Xa y los inhibidores directos de la trombina. Sin embargo, estos nuevos medicamentos sólo han sido aprobados en algunas indicaciones específicas y el resto de patologías aún requieren del uso de los anticoagulantes tradicionales, en especial antagonistas de la vitamina K (4).

La warfarina se descubrió a principios de los años 40 y es el anticoagulante oral más ampliamente usado en el mundo. Aunque se trata de un anticoagulante muy eficaz, su uso en la práctica clínica se ve limitado por sus estrechos márgenes de seguridad y toxicidad, importantes interacciones farmacológicas, fluctuaciones con la dieta, con factores fisio- lógicos, genéticos y los factores específicos del paciente (adherencia y seguimiento). Para garantizar su seguridad y uso efectivo en la práctica clínica, los antagonistas de la vitamina $\mathrm{K}$ requieren frecuente vigilancia de laboratorio y ajuste de la dosis (3). Algunos estudios han sugerido que los pacientes que toman warfarina están fuera de su rango terapéutico hasta $50 \%$ del tiempo. Un reciente metaanálisis de 71000 pacientes anticoagulados determinó que 44\% de eventos hemorrágicos ocurrieron con INR por encima del rango terapéutico, mientras que $48 \%$ de los eventos tromboembólicos se produjeron con INR por debajo del rango. Por esta razón, cualquier factor que mejore el tiempo en el que el paciente permanezca en rango terapéutico y así disminuir la frecuencia de eventos adversos debe ser una prioridad (4). Algunos estudios han evaluado los factores que mejoran los resultados en esta población de pacientes. Clínicas de anticoagulación, software computarizado para el seguimiento de INR y uso de automonitoreo de los valores de INR. En el caso de la anticoagulación se deben tener dos metas de tratamiento: aumento de tiempo en el rango terapéutico del INR (TRT) y reducción de los eventos adversos, tanto el sangrado como los episodios de retrombosis $(5,6)$.

El concepto de coordinar los servicios médicos, específicamente la terapia de anticoagulación oral fue desarrollado en Holanda siguiendo el modelo del profesor Jordan, quien en 1949 fundó el primer centro de trombosis en la Universidad de Utrecht. Muchas otras clínicas de anticoagulación fueron creadas en las siguientes décadas en los Países Bajos y actualmente existe un sistema nacional centralizado, de control ambulatorio de anticoagulación que cubre más de $90 \%$ del país. Hace cerca de 57 años Asken y Cherry establecen la triada esencial de componentes de una clínica de anticoagulación: un médico vigilante, un paciente cooperador y un labora-
Ver artículo: página 42

Dra. Ana C. Montenegro: Departamento de Medicina Interna, Clínica de Anticoagulación, Fundación Santa Fe de Bogotá; Dr. Andrés F. Buitrago: Departamento de Medicina Interna, Clínica de Anticoagulación Sección de Cardiología, Fundación Santa Fe de Bogotá. Bogotá, D.C. (Colombia).

E-mail: montenegroarenas@gmail. com 
torio confiable y rápidamente disponible (3).

En el año 1950 un grupo de internistas y cirujanos de la Universidad de Michigan desarrollaron una unidad especialmente dedicada al diagnóstico y tratamiento de las enfermedades tromboembólicas y propusieron la creación de programas de educación e investigación. Posteriormente en 1959 Servitt y Gallagher crearon una Clínica de Anticoagulación en Gran Bretaña y en 1989 se fundó la Federación Italiana de centros de anticoagulación. Este mismo año Landefeld y Rosenblatt publicaron que más de dos millones de americanos eran tratados con warfarina por múltiples patologías. El 1996 Rossendal concluye que la anticoagulación debe ser monitorizada por clínicas para minimizar riesgos, especialmente el sangrado y optimizar los beneficios de la terapia. Tanto la protección conferida por la warfarina como la aparición de eventos adversos secundarios están relacionados con un buen control de la terapia anticoagulante (6). El tiempo en el rango terapéutico es uno de los indicadores de la calidad del manejo de la anticoagulación oral con antagonistas de la vitamina K (4). El presente estudio realizado en una clínica de anticoagulación con 319 pacientes se evidenció un TRT de $52 \%$, con una tasa de sangrado mayor de $0.65 \%$ y sangrado menor de $5 \%$ en 14 meses de seguimiento. Estos porcentajes de sangrado son mucho menores comparados con la tasa de sangrados mayores y menores con warfarina en los estudios RELY y ARISTOTLE (alrededor de 3 y $15 \%$ respectivamente). Debido a que los estudios RELY y ARISTOTLE son estudios clínicos controlados y el estudio publicado en esta edición de la revista es un estudio descriptivo, éstos no pueden ser comparados directamente.

Como es bien sabido una de las grandes limitaciones de los estudios descriptivos es la pérdida de seguimiento de algunos pacientes, en el caso de la terapia anticoagulante ésta es una barrera muy importante en el adecuado manejo de los pacientes y en la obtención de TRT.

En conclusión el manejo de la anticoagulación en especial en pacientes manejados con antagonistas de la vitamina $\mathrm{K}$, debe ser sistemático, ordenado y debe incluir una adecuada educación del paciente, lo cual se logra efectivamente por medio de las clínicas de anticoagulación. Si bien en Colombia hay algunos estudios similares a éste, de carácter descriptivo en centros únicos, que ayudan a dar una aproximación al funcionamiento y beneficios de las clínicas, faltan estudios comparativos entre el manejo por parte de las clínicas versus el manejo tradicional con el fin de evaluar la real diferencia en la calidad, tasas de complicaciones, mortalidad y costoefectividad de la implementación de rutina de clínicas de anticoagulación en nuestro país.

\section{Referencias}

1. Bloomfield HE, Taylor BC, Krause A, Reddy P, Greer N, MacDonald R, Rutks I, Wilt TJ. Safe and Effective Anticoagulation in the Outpatient Setting: A Systematic Review of the Evidence. VA Evidence-based Synthesis Program (ESP) Center, Center for Chronic Disease Outcomes Research, Minneapolis VA Medical Center. 2011.

2. Isaza C, Beltrán L, Henao J, Porras G, Pinzón A, Vallejos A, Machado J. Factores genéticos y ambientales asociados con la respuesta a warfarina en pacientes colombianos. Biomedica 2010; 30(3): 410-20.

3. Rose AJ, Hylek EM, Ozonoff A, Ash AS, Reisman JI, Callahan PP, et al. Relevance of current guidelines for organizing an anticoagulation clinic. Am J Manag Care 2011; 17(4): 284-9.

4 Ageno W, Gallus AS, Wittkowsky A, Crowther M, Hylek EM, Palareti G. Oral anticoagulant therapy: Antithrombotic Therapy and Prevention of Thrombosis, 9th ed: American College of Chest Physicians Evidence-Based Clinical Practice Guidelines. Chest 2012; 141(2 Suppl): e44S-88S.

5. Macik BG. The future of anticoagulation clinics. J Thromb Thrombolysis 2003;16(1-2): 55-9.

6. Phillips KW,Ansell J. Outpatient management of oral vitamin $\mathrm{K}$ antagonist therapy: defining and measuring high-quality management. Expert Rev Cardiovasc Ther 2008; 6(1): 57-70.

7. Ocampo C, Hernández O, Velásquez CJ, Tobón I, Mejía FA. La clínica de anticoagulación del Hospital Universitario San Vicente de Paúl: demografía, efectividad y complicaciones. IATREIA 2004; 17(2): 105-114.

8. Miranda H, Osorio S, Giraldo DP, Duque J, Cataño JU, Tobón LI, et al. Tiempo en rango terapéutico en clínica de anticoagulación, reportes de eventos adversos y factores asociados a bajo TRT. Acta Med Colomb 2016; 41: 42-48. 